\title{
Isometric embeddings of polar Grassmannians and metric characterizations of their apartments
}

\author{
Mariusz Kwiatkowski $^{1}$ - Mark Pankov ${ }^{1}$
}

Received: 10 February 2015 / Accepted: 14 October 2016 / Published online: 28 November 2016

(C) The Author(s) 2016. This article is published with open access at Springerlink.com

\begin{abstract}
We describe isometric embeddings of polar Grassmann graphs formed by non-maximal singular subspaces. In almost all cases, they are induced by collinearitypreserving injections of polar spaces. As a simple consequence of this result, we get a metric characterization of apartments in polar Grassmannians.
\end{abstract}

Keywords Polar Grassmann graph · Apartment · Isometric embedding

Mathematics Subject Classification 51A50 - 51E24

\section{Introduction}

A building is a simplicial complex together with a distinguished family of subcomplexes, the so-called apartments, satisfying some axioms [20], see also [2]. All apartments are identified with a certain Coxeter system which defines the building type. The vertex set of the building is labeled by the nodes of the diagram of this Coxeter system. The set of all vertices corresponding to the same node is a building Grassmannian [13,18]. The intersections of apartments with Grassmannians are said to be apartments in these Grassmannians. Grassmannians have the natural adjacency relation coming from the building structure: two distinct vertices $a, b$ are adjacent if the building contains a simplex $P$ such that $P \cup\{a\}$ and $P \cup\{b\}$ are chambers, i.e.,

$凶 \quad$ Mariusz Kwiatkowski

mkw@matman.uwm.edu.pl

Mark Pankov

pankov@matman.uwm.edu.pl

1 Department of Mathematics and Computer Science, University of Warmia and Mazury, Słoneczna 54, Olsztyn, Poland 
maximal simplices in the building. In this case, we say that the line joining $a$ and $b$ is the set of all vertices $c$ for which $P \cup\{c\}$ is a chamber. So, every Grassmannian can be considered as a graph as well as a point-line geometry.

Every building of type $\mathrm{A}_{n}, n \geq 3$ is the flag complex of a certain $(n+1)$-dimensional vector space over a division ring, and the corresponding Grassmannians are formed by subspaces of the same dimension. Similarly, every building of type $C_{n}$ is the flag complex of a rank $n$ polar space and all buildings of type $\mathrm{D}_{n}$ can be obtained from polar spaces of type $\mathrm{D}_{n}$. The Grassmannians of such buildings are polar and half-spin Grassmannians. The associated graphs are said to be polar and half-spin Grassmann graphs. Note that polar Grassmann graphs formed by maximal singular subspaces are known as dual polar graphs. Classical Chow's theorems [3] (see also [9,13]) describe automorphisms of dual polar graphs and half-spin Grassmann graphs. The description of automorphisms of polar Grassmann graphs formed by non-maximal singular subspaces is given [13, Section 4.6]. In almost all cases, such automorphisms are induced by collineations of the associated polar spaces (except the case of polar and half-spin Grassmann graphs of type $D_{4}$ ).

In this paper isometric embeddings of polar Grassmann graphs will be investigated. Since graph isomorphisms can be characterized as surjective isometric embeddings, the results concerning automorphisms easily follow from the description of isometric embeddings.

Let $\Pi$ be a polar space of rank $n$. Denote by $\Gamma_{k}(\Pi)$ the polar Grassmann graph formed by $k$-dimensional singular subspaces of $\Pi$. Let also $\Pi^{\prime}$ be a polar space of rank $n^{\prime}$. By [14, Theorem 3], every isometric embedding of the dual polar graph $\Gamma_{n-1}(\Pi)$ in the dual polar graph $\Gamma_{n^{\prime}-1}\left(\Pi^{\prime}\right)$ is induced by a collinearity-preserving injection of $\Pi$ to the quotient polar space of $\Pi^{\prime}$ by a certain $\left(n^{\prime}-n-1\right)$-dimensional singular subspace. It follows from [14, Theorem 2] that apartments in the polar Grassmannian formed by maximal singular subspaces of $\Pi$ can be characterized as the images of isometric embeddings of the $n$-dimensional hypercube graph $H_{n}$ in $\Gamma_{n-1}(\Pi)$. If $\Pi$ and $\Pi^{\prime}$ are polar spaces of types $D_{n}$ and $D_{n^{\prime}}$ (respectively) and $n$ is even, then the same holds for isometric embeddings of the associated half-spin Grassmann graphs [16, Theorem 4]. By [16, Theorem 2], apartments in the half-spin Grassmannians of $\Pi$ can be characterized as the images of isometric embeddings of the half-cube graph $\frac{1}{2} H_{n}$ in the corresponding half-spin Grassmann graphs; as above, we assume that $n$ is even. Also, there is the following conjecture [16, Section 6]: if $n$ is odd, then there exist isometric embeddings of $\frac{1}{2} H_{n}$ in the half-spin Grassmann graphs of $\Pi$ whose images are not apartments.

In this paper similar results will be established for isometric embeddings of polar Grassmann graphs formed by non-maximal singular subspaces (Theorems 1-3). Our arguments are different from the arguments given in $[14,16]$. In dual polar graphs and half-spin Grassmann graphs the distance between two vertices is completely defined by the dimension of the intersection of the corresponding maximal singular subspaces. For polar Grassmann graphs formed by non-maximal singular subspaces the distance formula is more complicated (Sect. 2.4).

As a simple consequence of the main results, we get the following metric characterization of apartments in polar Grassmannians (Corollary 1): if $\Gamma_{k}(n)$ denotes the 
restriction of the graph $\Gamma_{k}(\Pi)$ to any apartment, then the image of every isometric embedding of $\Gamma_{k}(n)$ in $\Gamma_{k}(\Pi)$ is an apartment.

It must be pointed out that there is no similar characterization for apartments in Grassmannians of vector spaces. Let $V$ be an $n$-dimensional vector space (over a division ring). Consider the Grassmann graph $\Gamma_{k}(V)$ formed by $k$-dimensional subspaces of $V$. The restriction of $\Gamma_{k}(V)$ to every apartment of the corresponding Grassmannian is isomorphic to the Johnson graph $J(n, k)$. The image of every isometric embedding of $J(n, k)$ in $\Gamma_{k}(V)$ is an apartment only in the case when $n=2 k$. The images of all possible isometric embeddings of Johnson graphs in Grassmann graphs are described in [17, Chapter 4]. Also, [17, Chapter 3] contains the complete description of isometric embeddings of Grassmann graphs. They are defined by semilinear embeddings of special type; such semilinear embeddings are not necessarily strong.

Other characterizations of apartments in building Grassmannians can be found in $[4,7,10-12,15]$. Most of them are in terms of independent subsets of point-line geometries.

A building Grassmannian can be contained in other building Grassmannian as a subspace (in the sense of point-line geometry). There is a natural question: is it possible to determine all such subspaces? This problem is closely related to characterizing apartments and solved for some special cases [1,4-6]. For example, subspaces of polar Grassmannians isomorphic to Grassmannians of vector spaces are described in [1]. There is a similar description for subspaces of symplectic Grassmannians isomorphic to other symplectic Grassmannians [6].

\section{Basic notions and constructions}

\subsection{Graphs}

We will consider connected simple graphs only. In such a graph the distance $d(x, y)$ between two vertices $x, y$ is defined as the smallest number $i$ such that there is a path consisting of $i$ edges and connecting $x$ and $y$ [8, Section 15.1]. A path between $x$ and $y$ is said to be a geodesic if it is formed by precisely $d(x, y)$ edges. The graph diameter is the greatest distance between two vertices.

A clique is a subset in the vertex set of a graph, where any two distinct elements are adjacent vertices. Using Zorn lemma, we show that every clique is contained in a certain maximal clique.

An embedding of a graph $\Gamma$ in a graph $\Gamma^{\prime}$ is an injection of the vertex set of $\Gamma$ to the vertex set of $\Gamma^{\prime}$ transferring adjacent and non-adjacent vertices of $\Gamma$ to adjacent and non-adjacent vertices of $\Gamma^{\prime}$, respectively. Surjective embeddings are isomorphisms. Every embedding $f$ sends maximal cliques of $\Gamma$ to cliques of $\Gamma^{\prime}$ which are not necessarily maximal, i.e., subsets of maximal cliques. For any distinct maximal cliques $\mathcal{X}$ and $\mathcal{Y}$ of $\Gamma$ there exist non-adjacent vertices $x \in \mathcal{X}$ and $y \in \mathcal{Y}$. Then $f(x)$ and $f(y)$ are non-adjacent vertices of $\Gamma^{\prime}$ and there is no clique containing both $f(\mathcal{X})$ and $f(\mathcal{Y})$. So, we come to the following observation: every embedding transfers distinct maximal cliques to subsets of distinct maximal cliques.

An embedding is isometric if it preserves the distance between vertices. 


\subsection{Polar spaces}

A partial linear space is a pair $\Pi=(P, \mathcal{L})$, where $P$ is a non-empty set whose elements are called points and $\mathcal{L}$ is a family of proper subsets of $P$ called lines. Every line contains at least two points, and every point belongs to a certain line. Also, for any two distinct points there is at most one line containing them. The points are said to be collinear if such a line exists. A subspace of $\Pi$ is a subset $S \subset P$ such that for any two collinear points of $S$ the line joining them is contained in $S$. A subspace is called singular if any two distinct points of this subspace are collinear. The empty set, one-point sets and lines are singular subspaces. Using Zorn lemma, we establish that every singular subspace is contained in a maximal singular subspace. Two subspaces are called incident if one of them is contained in the other.

By $[2,13,19,21]$, a polar space is a partial linear space satisfying the following axioms:

(P1) Every line contains at least three points,

(P2) There is no point collinear to all points,

(P3) For every point and every line the point is collinear to one or all points of the line,

(P4) Any chain of mutually distinct incident singular subspaces is finite.

If a polar space has a singular subspace containing more than one line, then all maximal singular subspaces are projective spaces of the same dimension $n \geq 2$ and the number $n+1$ is called the rank of this polar space. Polar spaces of rank 2 (all maximal singular subspaces are lines) are known as generalized quadrangles. In the case when the rank of a polar space is greater than 2 every singular subspace is a subspace of a certain projective space and its dimension is defined.

Two polar spaces $\Pi=(P, \mathcal{L})$ and $\Pi^{\prime}=\left(P^{\prime}, \mathcal{L}^{\prime}\right)$ are isomorphic if there is a collineation of $\Pi$ to $\Pi^{\prime}$, i.e., a bijection $\alpha: P \rightarrow P^{\prime}$ such that $\alpha(\mathcal{L})=\mathcal{L}^{\prime}$.

All polar spaces of rank $\geq 3$ are known [20]. For example, there are polar spaces related to non-degenerate reflexive forms (alternating, symmetric and Hermitian). If such a form is trace-valued and has isotropic subspaces of dimension at least 2, then it defines a polar space: the point set is formed by all one-dimensional isotropic subspaces, the lines are defined by two-dimensional isotropic subspaces, and other isotropic subspaces correspond to singular subspaces of dimension greater than 1 .

Consider the $(2 n)$-element set $J:=\{ \pm 1, \ldots, \pm n\}$ and the partial linear space $\Pi_{n}$ whose point set is $J$ and whose lines are two-element subsets $\{i, j\}$ such that $j \neq-i$. Then $S \subset J$ is a singular subspace of $\Pi_{n}$ if and only if for every $i \in S$ we have $-i \notin S$. A singular subspace is maximal if it consists of $n$ points. The dimension of a singular subspace $S$ is equal to $|S|-1$, and maximal singular subspaces of $\Pi_{n}$ are $(n-1)$-dimensional. The partial linear space $\Pi_{n}$ satisfies the axioms (P2)-(P4), and we say that every partial linear space isomorphic to $\Pi_{n}$ is a thin polar space of rank $n$.

Let $\Pi=(P, \mathcal{L})$ be a polar space of rank $n$. For every subset $X \subset P$ the subspace of $\Pi$ spanned by $X$, i.e., the minimal subspace containing $X$, is denoted by $\langle X\rangle$. If any two distinct points of $X$ are collinear, then this subspace is singular. If a point is 
collinear to every point of $X$, then this point is collinear to all points of the subspace $\langle X\rangle$. A subset of $P$ consisting of $2 n$ distinct points $p_{1}, \ldots, p_{2 n}$ is a frame of $\Pi$ if for every $i$ there is unique $\sigma(i)$ such that $p_{i}$ and $p_{\sigma(i)}$ are non-collinear. Any $k$ distinct mutually collinear points in a frame span a $(k-1)$-dimensional singular subspace. We will use the following remarkable property of frames: for any two singular subspaces there is a frame such that these subspaces are spanned by subsets of the frame. Note that a thin polar space contains the unique frame which coincides with the set of points.

Every rank $n$ polar space satisfies one of the following conditions:

$\left(\mathrm{C}_{n}\right)$ Every $(n-2)$-dimensional singular subspace is contained in at least three maximal singular subspaces,

$\left(\mathrm{D}_{n}\right)$ Every $(n-2)$-dimensional singular subspace is contained in precisely two maximal singular subspaces.

We say that a polar space is of type $\mathrm{C}_{n}$ or $\mathrm{D}_{n}$ if the corresponding possibility is realized. For example, if a rank $n$ polar space is defined by an alternating or Hermitian form, then it is of type $C_{n}$. A thin polar space of rank $n$ is of type $D_{n}$. Other polar spaces of this type will be considered in Sect. 2.5.

\subsection{Polar Grassmannians}

Let $\Pi=(P, \mathcal{L})$ be a polar space or a thin polar space of rank $n$. For every $k \in\{0,1, \ldots, n-1\}$ we denote by $\mathcal{G}_{k}(\Pi)$ the polar Grassmannian consisting of $k$ dimensional singular subspaces of $\Pi$. Note that $\mathcal{G}_{0}(\Pi)$ coincides with $P$ and $\mathcal{G}_{n-1}(\Pi)$ is formed by maximal singular subspaces.

The polar Grassmann graph $\Gamma_{k}(\Pi)$ is the graph whose vertex set is $\mathcal{G}_{k}(\Pi)$. In the case when $k \leq n-2$ two distinct elements of $\mathcal{G}_{k}(\Pi)$ are adjacent vertices of $\Gamma_{k}(\Pi)$ if there is a $(k+1)$-dimensional singular subspace containing them. Two distinct maximal singular subspaces are adjacent vertices of $\Gamma_{n-1}(\Pi)$ if their intersection is $(n-2)$-dimensional. The graph $\Gamma_{n-1}(\Pi)$ is known as the dual polar graph associated with $\Pi$. If $\Pi$ is a thin polar space, then we write $\Gamma_{k}(n)$ instead of $\Gamma_{k}(\Pi)$. Note that $\Gamma_{n-1}(n)$ is isomorphic to the $n$-dimensional hypercube graph $H_{n}$.

For every frame of $\Pi$ the set consisting of all $k$-dimensional singular subspaces spanned by subsets of the frame is called the apartment of $\mathcal{G}_{k}(\Pi)$ associated with this frame. The restriction of the graph $\Gamma_{k}(\Pi)$ to every apartment of $\mathcal{G}_{k}(\Pi)$ is isomorphic to $\Gamma_{k}(n)$. By the frame property given in the previous subsection, for any two elements of $\mathcal{G}_{k}(\Pi)$ there is an apartment containing them. If $\Pi$ is a thin polar space, then there is the unique apartment of $\mathcal{G}_{k}(\Pi)$ which coincides with the polar Grassmannian.

For every singular subspace $S$ we denote by $[S\rangle_{k}$ the set of all $k$-dimensional singular subspaces containing $S$. This set is non-empty only in the case when the dimension of $S$ is not greater than $k$. Every subset of type

$$
[S\rangle_{n-1}, \quad S \in \mathcal{G}_{n-2}(\Pi)
$$

is called a line of $\mathcal{G}_{n-1}(\Pi)$. Each maximal clique in the dual polar graph $\Gamma_{n-1}(\Pi)$ is a line. 
Now we suppose that $k \leq n-2$. Let $S$ and $U$ be a pair of incident singular subspaces such that $\operatorname{dim} S \leq k \leq \operatorname{dim} U$. Denote by $[S, U]_{k}$ the set of all $X \in \mathcal{G}_{k}(\Pi)$ satisfying $S \subset X \subset U$. In the case when $S=\emptyset$ we write $\langle U]_{k}$ instead of $[S, U]_{k}$. If

$$
\operatorname{dim} S=k-1 \text { and } \operatorname{dim} U=k+1,
$$

then $[S, U]_{k}$ is called a line of $\mathcal{G}_{k}(\Pi)$. In the case when $k=0$ we get a line of $\Pi$.

If $1 \leq k \leq n-3$, then there are precisely the following two types of maximal cliques of $\Gamma_{k}(\Pi)$ :

- The $\operatorname{star}[S, U]_{k}, S \in \mathcal{G}_{k-1}(\Pi)$ and $U \in \mathcal{G}_{n-1}(\Pi)$;

- The top $\langle U]_{k}, U \in \mathcal{G}_{k+1}(\Pi)$.

Every star of $\mathcal{G}_{n-2}(\Pi)$ is a line contained in a certain top, and all maximal cliques of $\Gamma_{n-2}(\Pi)$ are tops. Tops and stars of $\mathcal{G}_{0}(\Pi)=P$ are lines and maximal singular subspaces of $\Pi$, respectively.

In the case when $1 \leq k \leq n-2$ every subset of type

$$
[S\rangle_{k}, \quad S \in \mathcal{G}_{k-1}(\Pi)
$$

is said to be a big star. Every big $\operatorname{star}[S\rangle_{k}$ (together with all lines of $\mathcal{G}_{k}(\Pi)$ contained in it) is a polar space of rank $n-k$. We denote this polar space by $\Pi_{S}$. Every $i$-dimensional singular subspace of $\Pi_{S}$ is a subset of type $[S, U]_{k}$, where $U$ is a $(k+i)$-dimensional singular subspace containing $S$. Therefore, for every $i \in\{0, \ldots, n-k-1\}$ the Grassmannian $\mathcal{G}_{i}\left(\Pi_{S}\right)$ can be naturally identified with the set $[S\rangle_{k+i}$ and the polar Grassmann graph $\Gamma_{i}\left(\Pi_{S}\right)$ coincides with the restriction of the graph $\Gamma_{k+i}(\Pi)$ to $[S\rangle_{k+i}$. If $\mathcal{A}$ is an apartment of $\mathcal{G}_{k}(\Pi)$ such that $S$ is spanned by a subset of the frame associated with $\mathcal{A}$, then $\mathcal{A} \cap[S\rangle_{k}$ is a frame of $\Pi_{S}$. Conversely, every frame of $\Pi_{S}$ can be obtained in this way. Similarly, every apartment of $\mathcal{G}_{i}\left(\Pi_{S}\right)$ is the intersection of $[S\rangle_{k+i}$ and an apartment of $\mathcal{G}_{k+i}(\Pi)$ such that $S$ is spanned by a subset of the associated frame.

\subsection{Distance in polar Grassmann graphs}

The Grassmann graph $\Gamma_{k}(\Pi)$ is connected for every $k$. The distance between $X, Y \in$ $\mathcal{G}_{n-1}(\Pi)$ in the dual polar graph $\Gamma_{n-1}(\Pi)$ is

$$
n-1-\operatorname{dim}(X \cap Y) .
$$

In particular, the diameter of $\Gamma_{n-1}(\Pi)$ is equal to $n$ (the dimension of the empty set is $-1)$.

Let $X, Y \in \mathcal{G}_{k}(\Pi)$ and $k \leq n-2$. It is clear that the distance $d(X, Y)$ between $X$ and $Y$ in the graph $\Gamma_{k}(\Pi)$ is not less than $k-\operatorname{dim}(X \cap Y)$.

Suppose that there is a point $p \in X \backslash Y$ collinear to all points of $Y$. Then there exists a point $q \in Y \backslash X$ collinear to all points of $X$. This follows, for example, from the existence of a frame of $\Pi$ containing the point $p$ and such that $X$ and $Y$ are spanned 
by subsets of this frame. If $X \cap Y$ is $(k-1)$-dimensional, then $X$ and $Y$ are adjacent vertices of $\Gamma_{k}(\Pi)$. In the case when

$$
\operatorname{dim}(X \cap Y) \leq k-2
$$

we take any $k$-dimensional singular subspace $X_{1}$ spanned by the point $q$ and a $(k-1)$ dimensional subspace of $X$ containing $X \cap Y$ and $p$. Then $X$ and $X_{1}$ are adjacent vertices of $\Gamma_{k}(\Pi)$ and

$$
\operatorname{dim}\left(X_{1} \cap Y\right)=\operatorname{dim}(X \cap Y)+1 .
$$

Note that $p$ is a point of $X_{1} \backslash Y$ collinear to all points of $Y$. Step by step, we construct a path

$$
X, X_{1}, \ldots, X_{i}=Y, \quad i=k-\operatorname{dim}(X \cap Y)
$$

in the graph $\Gamma_{k}(\Pi)$ which means that

$$
d(X, Y)=k-\operatorname{dim}(X \cap Y) .
$$

Now we suppose that every point of $X \backslash Y$ is non-collinear to a certain point of $Y$. Then every point of $Y \backslash X$ is non-collinear to a certain point of $X$. We take any frame whose subsets span $X$ and $Y$ and construct a $k$-dimensional singular subspace $X^{\prime}$ satisfying the following conditions:

(1) $X$ and $X^{\prime}$ are adjacent vertices of $\Gamma_{k}(\Pi)$,

(2) There is a point of $X^{\prime} \backslash Y$ collinear to all points of $Y$,

(3) $X \cap Y=X^{\prime} \cap Y$.

Then

$$
d\left(X^{\prime}, Y\right)=k-\operatorname{dim}\left(X^{\prime} \cap Y\right)=k-\operatorname{dim}(X \cap Y) .
$$

We have

$$
d(X, Y)=k-\operatorname{dim}(X \cap Y)+1,
$$

since there is no vertex of $\Gamma_{k}(\Pi)$ adjacent to $X$ and intersecting $Y$ in a subspace of dimension greater than the dimension of $X \cap Y$.

So, if $k \leq n-2$, then the diameter of $\Gamma_{k}(\Pi)$ is equal to $k+2$ and we have the following description of the distance.

Lemma 1 Let $X, Y \in \mathcal{G}_{k}(\Pi)$ and $k \leq n-2$. The distance between $X$ and $Y$ in $\Gamma_{k}(\Pi)$ is equal to $m$ if and only if one of the following possibilities is realized:

(1) $\operatorname{dim}(X \cap Y)=k-m$, there is a point of $X \backslash Y$ collinear to all points of $Y$ and there is a point of $Y \backslash X$ collinear to all points of $X$; 
(2) $m>1, \operatorname{dim}(X \cap Y)=k-m+1$, every point of $X \backslash Y$ is non-collinear to a certain point of $Y$ and every point of $Y \backslash X$ is non-collinear to a certain point of $X$.

If $m=k+2$, then only the second possibility is realized.

\subsection{Polar spaces of type $D_{n}$ and half-spin Grassmannians}

It was noted above that a thin polar space of rank $n$ is of type $D_{n}$. Let $V$ be a $(2 n)$ dimensional vector space over a field. If the characteristic of this field is not equal to 2 and there is a non-degenerate symmetric bilinear form on $V$ whose maximal isotropic subspaces are $n$-dimensional, then the associated polar space is of type $D_{n}$. In the case when the characteristic of the field is equal to 2 we consider a non-defect quadratic form on $V$ such that maximal singular subspaces are $n$-dimensional. The associated polar space (the points are one-dimensional singular subspaces, and the lines are defined by two-dimensional singular subspaces) is also of type $D_{n}$. It follows from Tits's description of polar spaces [20] that every polar space of type $\mathrm{D}_{n}, n \geq 4$ is isomorphic to one of the polar spaces mentioned above.

Let $\Pi=(P, \mathcal{L})$ be a polar space of type $\mathrm{D}_{n}$ (possibly thin) and $n \geq 4$. Then $\mathcal{G}_{n-1}(\Pi)$ can be uniquely decomposed in the sum of two disjoint subsets $\mathcal{G}_{+}(\Pi)$ and $\mathcal{G}_{-}(\Pi)$ such that the distance between any two elements of $\mathcal{G}_{\delta}(\Pi), \delta \in\{+,-\}$ in the dual polar graph $\Gamma_{n-1}(\Pi)$ is even and the same distance between any $S \in \mathcal{G}_{+}(\Pi)$ and $U \in \mathcal{G}_{-}(\Pi)$ is odd. These subsets are known as the half-spin Grassmannians.

If $\Pi$ is defined by a non-degenerate symmetric bilinear form $\Omega$, then the maximal singular subspaces of $\Pi$ are identified with the maximal isotropic subspaces of $\Omega$ and the half-spin Grassmannians are the orbits of the action of the group $\mathrm{O}_{+}(\Omega)$ (the orthogonal group formed by elements with determinant 1) on the set of all maximal isotropic subspaces. Every element of $\mathrm{O}(\Omega) \backslash \mathrm{O}_{+}(\Omega)$ induces a collineation of $\Pi$ which maps one of the half-spin Grassmannians to the other. The same holds for the case when $\Pi$ is defined by a quadratic form. So, collineations of $\Pi$ sending $\mathcal{G}_{+}(\Pi)$ to $\mathcal{G}_{-}(\Pi)$ always exist.

Suppose that $n=4$ and $\delta \in\{+,-\}$. For every line $L \in \mathcal{L}$, the set $[L\rangle_{\delta}$ consisting of all elements of $\mathcal{G}_{\delta}(\Pi)$ containing $L$ is called a line of $\mathcal{G}_{\delta}(\Pi)$. The half-spin Grassmannian $\mathcal{G}_{\delta}(\Pi)$ together with the family of all such lines is a polar space of type $D_{4}$. We denote this polar space by $\Pi_{\delta}$. The polar spaces $\Pi_{+}$and $\Pi_{-}$are isomorphic (every collineation of $\Pi$ transferring $\mathcal{G}_{+}(\Pi)$ to $\mathcal{G}_{-}(\Pi)$ induces a collineation between these polar spaces). The half-spin Grassmannians corresponding to $\Pi_{\delta}$ are the point set $P$ and $\mathcal{G}_{-\delta}(\Pi)$, where $-\delta$ is the complement of $\delta$ in the set $\{+,-\}$. The associated polar spaces are $\Pi$ and $\Pi_{-\delta}$. Therefore, $\Pi$ is isomorphic to both $\Pi_{+}$and $\Pi_{-}$. Since there is a natural one-to-one correspondence between lines of the polar spaces $\Pi$ and $\Pi_{\delta}$, every collineation from $\Pi$ to $\Pi_{\delta}$ induces a bijective transformation of $\mathcal{G}_{1}(\Pi)$. This transformation is an automorphism of the graph $\Gamma_{1}(\Pi)$.

Let $\alpha$ be a collineation from $\Pi$ to $\Pi_{-\delta}$. It induces collineations of the polar spaces $\Pi_{+}$and $\Pi_{-}$to the polar spaces associated with the half-spin Grassmannians of $\Pi_{-\delta}$. So, we get a collineation of $\Pi_{\delta}$ to $\Pi$ or $\Pi_{\delta}$. Since there are collineations of $\Pi_{-\delta}$ transferring $\Pi$ to $\Pi_{\delta}$, we can suppose that $\alpha$ induces a collineation of $\Pi_{\delta}$ to itself. 


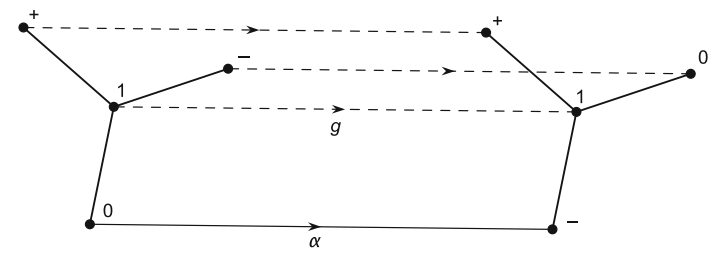

The automorphism $g$ of $\Gamma_{1}(\Pi)$ induced by $\alpha$ has the following properties:

- If $U \in \mathcal{G}_{\delta}(\Pi)$, then $g\left([p, U]_{1}\right)$ is a top for every point $p \in U$,

- If $U \in \mathcal{G}_{-\delta}(\Pi)$, then there exists $U^{\prime} \in \mathcal{G}_{-\delta}(\Pi)$ such that every $g\left([p, U]_{1}\right)$ is a star contained in $\left\langle U^{\prime}\right]_{1}$.

See [13, Section 4.6] for the details.

\section{Main results}

From this moment we suppose that $\Pi=(P, \mathcal{L})$ is a polar space or a thin polar space of rank $n$ and $\Pi^{\prime}=\left(P^{\prime}, \mathcal{L}^{\prime}\right)$ is a polar space of rank $n^{\prime}$.

Let $f: P \rightarrow P^{\prime}$ be a collinearity-preserving injection, i.e., $f$ sends collinear and non-collinear points of $\Pi$ to collinear and non-collinear points of $\Pi^{\prime}$, respectively. We now show that $f$ transfers every frame of $\Pi$ to a subset in a frame of $\Pi^{\prime}$.

If $\mathcal{F}$ is a frame of $\Pi$, then for every point $p \in f(\mathcal{F})$ there is a unique point of $f(\mathcal{F})$ non-collinear to $p$. This means that $n \leq n^{\prime}$ and $f(\mathcal{F})$ is a frame of $\Pi^{\prime}$ if $n=n^{\prime}$. In the case when $n^{\prime}>n$ we consider the set formed by all points of $\Pi^{\prime}$ collinear with all points of $f(\mathcal{F})$. If $n^{\prime}-n \geq 2$, then this is a polar space of rank $n^{\prime}-n$ and $f(\mathcal{F})$ together with any frame of this polar space gives a frame of $\Pi^{\prime}$. If $n^{\prime}-n=1$, then our set consists of mutually non-collinear points and $f(\mathcal{F})$ together with any pair of such points defines a frame of $\Pi^{\prime}$.

Since every singular subspace $S$ of $\Pi$ is spanned by a subset of a certain frame of $\Pi$, the dimension of the singular subspace $\langle f(S)\rangle$ is equal to the dimension of $S$. It is clear that $f$ is an isometric embedding of $\Gamma_{0}(\Pi)$ in $\Gamma_{0}\left(\Pi^{\prime}\right)$ and for every $k \in\{1, \ldots, n-1\}$ the mapping

$$
\begin{aligned}
& (f)_{k}: \mathcal{G}_{k}(\Pi) \rightarrow \mathcal{G}_{k}\left(\Pi^{\prime}\right) \\
& S \rightarrow\langle f(S)\rangle
\end{aligned}
$$

is an isometric embedding of $\Gamma_{k}(\Pi)$ in $\Gamma_{k}\left(\Pi^{\prime}\right)$. If $n=n^{\prime}$ and $\Pi$ is a thin polar space, then the image of this mapping is an apartment of $\mathcal{G}_{k}\left(\Pi^{\prime}\right)$.

Now we suppose that $n \leq n^{\prime}$ and take any $m$-dimensional singular subspace $S$ of $\Pi^{\prime}$ such that

$$
m \leq n^{\prime}-n-1
$$

(this subspace is empty if $n=n^{\prime}$ ). Then $\Pi_{S}^{\prime}$ is a polar space of rank $n^{\prime}-m-1 \geq n$ (in the case when $S$ is empty, this polar space coincides with $\Pi^{\prime}$ ). Every collinearitypreserving injection of $\Pi$ to $\Pi_{S}^{\prime}$ induces an isometric embedding of $\Gamma_{k}(\Pi)$ in $\Gamma_{k}\left(\Pi_{S}^{\prime}\right)$. 
For every $k \in\{0,1, \ldots, n-1\}$ this mapping can be considered as an isometric embedding of $\Gamma_{k}(\Pi)$ in $\Gamma_{k^{\prime}}\left(\Pi^{\prime}\right)$, where $k^{\prime}=m+k+1$.

Remark 1 Suppose that the polar spaces $\Pi$ and $\Pi^{\prime}$ are embedded in the projective spaces associated with vector spaces $V$ and $V^{\prime}$, respectively. Every collineation from $\Pi$ to $\Pi^{\prime}$ is induced by a semilinear isomorphism of $V$ to $V^{\prime}$ [9, Chapter III]. Similarly, every collinearity-preserving mapping from $\Pi$ to $\Pi^{\prime}$ can be obtained from a certain semilinear mapping $l: V \rightarrow V^{\prime}$ (the proof is a modification of the proof given in $[9$, Chapter III]). Note that the homomorphism between division rings associated with $l$ is not necessarily surjective. In the general case, we cannot state that $l$ is a strong semilinear embedding (a semilinear injection transferring any collection of linearly independent vectors to linearly independent vectors). However, this statement is obvious if our polar spaces are of type $D_{n}$ or for the symplectic polar spaces.

In this paper we will investigate isometric embeddings of the polar Grassmann graph $\Gamma_{k}(\Pi)$ (this graph coincides with $\Gamma_{k}(n)$ if $\Pi$ is a thin polar space) in the polar Grassmann graph $\Gamma_{k^{\prime}}\left(\Pi^{\prime}\right)$. We start from the following simple observation that will be proved in Sect. 4 .

Proposition 1 If $n \geq 4$ and $f$ is an isometric embedding of $\Gamma_{0}(\Pi)$ in $\Gamma_{m}\left(\Pi^{\prime}\right)$, then $m \leq n^{\prime}-n$ and there is an $(m-1)$-dimensional singular subspace $S$ in $\Pi^{\prime}$ such that the image of $f$ is contained in $[S\rangle_{m}$ and $f$ is a collinearity-preserving injection of $\Pi$ to $\Pi_{S}^{\prime}$.

Remark 2 The diameter of $\Gamma_{0}(\Pi)$ is equal to 2 , and every embedding of this graph is isometric.

All isometric embeddings of the dual polar graph $\Gamma_{n-1}(\Pi)$ in the dual polar graph $\Gamma_{n^{\prime}-1}\left(\Pi^{\prime}\right)$ are described in [14]. The existence of such embeddings implies that the diameter of $\Gamma_{n-1}(\Pi)$ is not greater than the diameter of $\Gamma_{n^{\prime}-1}\left(\Pi^{\prime}\right)$, i.e., $n \leq n^{\prime}$. By [14, Theorem 2], the image of every isometric embedding of the $n$-dimensional hypercube graph $H_{n}=\Gamma_{n-1}(n)$ in the dual polar graph $\Gamma_{n^{\prime}-1}\left(\Pi^{\prime}\right)$ is an apartment of $\mathcal{G}_{n-1}\left(\Pi_{S}^{\prime}\right)$, where $S$ is an $\left(n^{\prime}-n-1\right)$-dimensional singular subspace of $\Pi^{\prime}$. Using this result and [13, Theorem 4.17] the author shows that every isometric embedding of $\Gamma_{n-1}(\Pi)$ in $\Gamma_{n^{\prime}-1}\left(\Pi^{\prime}\right)$ is induced by a collinearity-preserving injection from $\Pi$ to $\Pi_{S}^{\prime}$, where, as above, $S$ is an $\left(n^{\prime}-n-1\right)$-dimensional singular subspace of $\Pi^{\prime}$ [14, Theorem 3].

We will consider the case when our polar Grassmann graphs both are formed by non-maximal singular subspaces. The first result concerns the case when $n \geq 5$ and $1 \leq k \leq n-4$.

Theorem 1 Suppose that $n \geq 5$. If $f$ is an isometric embedding of $\Gamma_{k}(\Pi)$ in $\Gamma_{k^{\prime}}\left(\Pi^{\prime}\right)$ and $1 \leq k \leq n-4$, then

$$
k \leq k^{\prime}, \quad n-k \leq n^{\prime}-k^{\prime}
$$

and there is a $\left(k^{\prime}-k-1\right)$-dimensional singular subspace $S$ of $\Pi^{\prime}$ such that the image of $f$ is contained in $[S\rangle_{k^{\prime}}$ and $f$ is induced by a collinearity-preserving injection of $\Pi$ to $\Pi_{S}^{\prime}$. 
It must be pointed out that in Theorem 1 there is no assumption concerning $n^{\prime}$ and $k^{\prime}$. The case when $n \geq 4$ and $k=n-3$ is different.

Theorem 2 Suppose that $n \geq 4$ and $f$ is an isometric embedding of $\Gamma_{n-3}(\Pi)$ in $\Gamma_{k^{\prime}}\left(\Pi^{\prime}\right)$. If $\Pi$ is a polar space of type $\mathrm{C}_{n}$, then

$$
n-3 \leq k^{\prime} \leq n^{\prime}-3
$$

and there is a $\left(k^{\prime}-n+2\right)$-dimensional singular subspace $S$ of $\Pi^{\prime}$ such that the image of $f$ is contained in $[S\rangle_{k^{\prime}}$ and $f$ is induced by a collinearity-preserving injection of $\Pi$ to $\Pi_{S}^{\prime}$.

In the case when $\Pi$ is a polar space of type $D_{n}$ the following assertions are fulfilled:

(1) If $n=4$, then $1 \leq k^{\prime} \leq n^{\prime}-3$ and there is a $\left(k^{\prime}-2\right)$-dimensional singular subspace $S$ of $\Pi^{\prime}$ such that the image of $f$ is contained in $[S\rangle_{k^{\prime}}$. Also, there is an automorphism $g$ of $\Gamma_{1}(\Pi)$ (possibly the identity) such that the composition $\mathrm{fg}$ is induced by a collinearity-preserving injection from $\Pi$ to $\Pi_{S}^{\prime}$.

(2) If $n \geq 5$ and $k^{\prime}=n^{\prime}-3$, then $n \leq n^{\prime}$ and there is a $\left(n^{\prime}-n-1\right)$-dimensional singular subspace $S$ of $\Pi^{\prime}$ such that the image of $f$ is contained in $[S\rangle_{k^{\prime}}$ and $f$ is induced by a collinearity-preserving injection from $\Pi$ to $\Pi_{S}^{\prime}$.

Theorem 2 does not contain any assumption concerning $n^{\prime}$ and $k^{\prime}$ except the case when $\Pi$ is a polar space of type $\mathrm{D}_{n}, n \geq 5$. In this special case we can describe isometric embeddings of $\Gamma_{n-3}(\Pi)$ in $\Gamma_{n^{\prime}-3}\left(\Pi^{\prime}\right)$ only (see Remark 3 for the reasons).

Our third result covers the case when $n=n^{\prime}$ and $k=k^{\prime}=n-2$.

Theorem 3 If $n=n^{\prime}$, then every isometric embedding of $\Gamma_{n-2}(\Pi)$ in $\Gamma_{n-2}\left(\Pi^{\prime}\right)$ is induced by a collinearity-preserving injection of $\Pi$ to $\Pi^{\prime}$.

We cannot prove the same statement for isometric embeddings of $\Gamma_{n-2}(\Pi)$ in $\Gamma_{n^{\prime}-2}\left(\Pi^{\prime}\right)$ if $n<n^{\prime}$ (see Remark 4 for the reasons).

As a direct consequence of the above results, we get the following characterization of apartments in polar Grassmannians.

Corollary 1 The image of every isometric embedding of $\Gamma_{k}(n)$ in $\Gamma_{k}(\Pi)$ is an apartment of $\mathcal{G}_{k}(\Pi)$.

Proof For $k=0$ the statement follows directly from the frame definition. The case $k=n-1$ was considered in [14, Theorem 2]. If $1 \leq k \leq n-2$, then we apply Theorems $1-3$ to isometric embeddings of $\Gamma_{k}(n)$ in $\Gamma_{k}(\Pi)$.

Also, Theorems 1 and 2 imply that for the residue $\Gamma_{k}\left(\Pi_{S}\right)$, where $S$ is a singular subspace, the same statement holds with some restrictions on $k^{\prime}$ and the type of $\Pi^{\prime}$.

\section{Proof of Proposition 1 and Theorem 1}

\subsection{Triangles}

We say that three distinct mutually adjacent vertices of $\Gamma_{k}(\Pi)$ form a triangle if they do not belong to a common line of $\mathcal{G}_{k}(\Pi)$. The existence of triangles implies that 
$k \leq n-2$. If $1 \leq k \leq n-3$, then there are the following two types of triangles: star-triangles contained in stars and top-triangles contained in tops [13, Lemma 4.10]. If $S_{1}, S_{2}, S_{3} \in \mathcal{G}_{k}(\Pi)$ form a star-triangle, then

$$
\operatorname{dim}\left(S_{1} \cap S_{2} \cap S_{3}\right)=k-1 \text { and } \operatorname{dim}\left\langle S_{1}, S_{2}, S_{3}\right\rangle=k+2
$$

In the case when $S_{1}, S_{2}, S_{3} \in \mathcal{G}_{k}(\Pi)$ form a top-triangle we have

$$
\operatorname{dim}\left(S_{1} \cap S_{2} \cap S_{3}\right)=k-2 \text { and } \operatorname{dim}\left\langle S_{1}, S_{2}, S_{3}\right\rangle=k+1 .
$$

Each triangle of $\Pi$ is a star-triangle, and $\mathcal{G}_{n-2}(\Pi)$ contains only top-triangles.

Lemma 2 If $n \geq 3$ and $k \leq n-3$, then every embedding of $\Gamma_{k}(\Pi)$ in $\Gamma_{k^{\prime}}\left(\Pi^{\prime}\right)$ transfers triangles to triangles.

Proof Let $f$ be an embedding of $\Gamma_{k}(\Pi)$ in $\Gamma_{k^{\prime}}\left(\Pi^{\prime}\right)$ and $k \leq n-3$. Suppose that $S_{1}, S_{2}, S_{3} \in \mathcal{G}_{k}(\Pi)$ form a triangle and $\mathcal{X}$ is a maximal clique of $\Gamma_{k}(\Pi)$ containing this triangle. We state that there is a maximal clique $\mathcal{Y}$ of $\Gamma_{k}(\Pi)$ intersecting $\mathcal{X}$ precisely in the line joining $S_{1}$ and $S_{2}$. Indeed, if $1 \leq k \leq n-3$, then maximal cliques of $\Gamma_{k}(\Pi)$ are stars and tops and the statement is obvious. Maximal cliques of $\Gamma_{0}(\Pi)$ are maximal singular subspaces of $\Pi$, and it is well known that for any singular subspace $S$ there exist maximal singular subspaces $M$ and $M^{\prime}$ such that $S=M \cap M^{\prime}$.

So, $S_{3} \notin \mathcal{Y}$ and $\mathcal{Y}$ contains a vertex $Y$ non-adjacent to $S_{3}$. Since $f(Y)$ is adjacent to $f\left(S_{1}\right)$ and $f\left(S_{2}\right)$, it is adjacent to all vertices of $\Gamma_{k^{\prime}}\left(\Pi^{\prime}\right)$ belonging to the line joining $f\left(S_{1}\right)$ and $f\left(S_{2}\right)$. On the other hand, $f(Y)$ and $f\left(S_{3}\right)$ are not adjacent. Therefore, $f\left(S_{3}\right)$ is not on this line.

Lemma 3 If $n \geq 3$ and $k \leq n-3$, then for any embedding of $\Gamma_{k}(\Pi)$ in $\Gamma_{k^{\prime}}\left(\Pi^{\prime}\right)$ the image of every maximal clique of $\Gamma_{k}(\Pi)$ cannot be contained in two maximal cliques of different types.

Proof In this case, every maximal clique of $\Gamma_{k}(\Pi)$ contains a triangle. On the other hand, the intersection of two maximal cliques of different types is empty or a line. Lemma 2 gives the claim.

\subsection{Proof of Proposition 1}

Suppose that $f$ is an embedding of $\Gamma_{0}(\Pi)$ in $\Gamma_{m}\left(\Pi^{\prime}\right)$ (see Remark 2) and $n \geq 4$.

Let $M$ be a maximal singular subspace of $\Pi$. We take any maximal singular subspace $M^{\prime}$ such that $M \cap M^{\prime}$ is $(n-2)$-dimensional. Consider two maximal cliques of $\Gamma_{m}\left(\Pi^{\prime}\right)$ containing $f(M)$ and $f\left(M^{\prime}\right)$. By the observation from Sect. 2.1, they are distinct. The intersection of these cliques contains more than one element. Moreover, this intersection is a line of $\mathcal{G}_{m}\left(\Pi^{\prime}\right)$ if one of the cliques is a top. Then $f\left(M \cap M^{\prime}\right)$ is contained in a line. The latter contradicts Lemma 2, since the dimension of $M \cap M^{\prime}$ is equal to $n-2 \geq 2$ and $\left\langle M \cap M^{\prime}\right]_{0}$ contains triangles. 
Thus $f(M)$ is contained in a star. Hence there exists $S \in \mathcal{G}_{m-1}\left(\Pi^{\prime}\right)$ such that

$$
f(M) \subset[S\rangle_{m}
$$

Let $p \in P \backslash M$. It follows from the axiom (P3) that the set of all points of $M$ collinear to $p$ is an $(n-2)$-dimensional singular subspace. Denote by $M^{\prime}$ the maximal singular subspace spanned by this subspace and the point $p$. As above, we obtain that

$$
f\left(M^{\prime}\right) \subset\left[S^{\prime}\right\rangle_{m}
$$

for a certain $S^{\prime} \in \mathcal{G}_{m-1}\left(\Pi^{\prime}\right)$. The singular subspace $M \cap M^{\prime}$ contains more than one point, and for any distinct points $t, q \in M \cap M^{\prime}$, we have

$$
S=f(t) \cap f(q)=S^{\prime}
$$

Therefore, $f(p) \in[S\rangle_{m}$ for every point $p \in P$.

So, the image of $f$ is contained in $[S\rangle_{m}$ and $f$ is a collinearity-preserving injection of $\Pi$ to $\Pi_{S}^{\prime}$. The rank of $\Pi_{S}^{\prime}$ is equal to $n^{\prime}-m$, and it is not less than $n$ which implies that $m \leq n^{\prime}-n$.

\subsection{Technical result}

Let $f$ be an isometric embedding of $\Gamma_{k}(\Pi)$ in $\Gamma_{k^{\prime}}\left(\Pi^{\prime}\right)$ and $1 \leq k \leq n-3$. Then maximal cliques of $\Gamma_{k}(\Pi)$ are stars and tops and there exist pairs of distinct maximal cliques whose intersections contain more than one element. In the case when $k^{\prime} \geq n^{\prime}-2$ there is only one type of maximal cliques in $\Gamma_{k^{\prime}}\left(\Pi^{\prime}\right)$ (tops if $k^{\prime}=n^{\prime}-2$ and lines if $\left.k^{\prime}=n^{\prime}-1\right)$ and the intersection of any two distinct maximal cliques contains at most one element. It was noted in Sect. 2.1 that $f$ sends distinct maximal cliques to subsets of distinct maximal cliques. This guarantees that $k^{\prime} \leq n^{\prime}-3$. Also, the existence of isometric embeddings of $\Gamma_{k}(\Pi)$ in $\Gamma_{k^{\prime}}\left(\Pi^{\prime}\right)$ implies that the diameter of $\Gamma_{k}(\Pi)$ is not greater than the diameter of $\Gamma_{k^{\prime}}\left(\Pi^{\prime}\right)$. By Sect. 2.4. the diameters of these graphs are equal to $k+2$ and $k^{\prime}+2$, respectively. Therefore, $k \leq k^{\prime}$.

Proposition 2 If $f$ transfers stars to subsets of stars, then

$$
n-k \leq n^{\prime}-k^{\prime}
$$

and there exists a $\left(k^{\prime}-k-1\right)$-dimensional singular subspace $S$ of $\Pi^{\prime}$ such that the image of $f$ is contained in $[S\rangle_{k^{\prime}}$ and $f$ is induced by a collinearity-preserving injection of $\Pi$ to $\Pi_{S}^{\prime}$.

Proposition 2 will be proved in several steps. Our first step is the following. 
Lemma $4 f$ transfers big stars to subsets of big stars.

Proof Let $S \in \mathcal{G}_{k-1}(\Pi)$. The big star $[S\rangle_{k}$ is the union of all stars $[S, U]_{k}$, where $U$ is a maximal singular subspace of $\Pi$ containing $S$. By our assumption, $f$ transfers stars $[S, X]_{k}$ and $[S, Y]_{k}$ to subsets contained in some stars $\left[S^{\prime}, X^{\prime}\right]_{k^{\prime}}$ and $\left[S^{\prime \prime}, Y^{\prime}\right]_{k^{\prime}}$, respectively. If $X$ and $Y$ are adjacent vertices of $\Gamma_{n-1}(\Pi)$, i.e., the dimension of $X \cap Y$ is equal to $n-2 \geq k+1$, then the intersection of $[S, X]_{k}$ and $[S, Y]_{k}$ contains more than one element and the same holds for the intersection of $\left[S^{\prime}, X^{\prime}\right]_{k^{\prime}}$ and $\left[S^{\prime \prime}, Y^{\prime}\right]_{k^{\prime}}$. The latter is possible only in the case when $S^{\prime}=S^{\prime \prime}$. Since the restriction of the graph $\Gamma_{n-1}(\Pi)$ to $[S\rangle_{n-1}$ is connected, there exists $S^{\prime} \in \mathcal{G}_{k^{\prime}-1}\left(\Pi^{\prime}\right)$ such that the images of all stars $[S, X]_{k}$ are contained in some stars of type $\left[S^{\prime}, X^{\prime}\right]_{k^{\prime}}$. This means that $f$ transfers the big star $[S\rangle_{k}$ to a subset of the big star $\left[S^{\prime}\right\rangle_{k^{\prime}}$.

The intersection of two distinct big stars contains at most one element. This implies that for any $S \in \mathcal{G}_{k-1}(\Pi)$ there is unique $S^{\prime} \in \mathcal{G}_{k^{\prime}-1}\left(\Pi^{\prime}\right)$ such that $f\left([S\rangle_{k}\right)$ is contained in $\left[S^{\prime}\right\rangle_{k^{\prime}}$. So, our embedding induces a mapping

$$
f_{k-1}: \mathcal{G}_{k-1}(\Pi) \rightarrow \mathcal{G}_{k^{\prime}-1}\left(\Pi^{\prime}\right)
$$

such that

$$
f\left([S\rangle_{k}\right) \subset\left[f_{k-1}(S)\right\rangle_{k^{\prime}}
$$

for every $S \in \mathcal{G}_{k-1}(\Pi)$.

Lemma 5 The mapping $f_{k-1}$ is injective.

Proof Let $S$ and $U$ be distinct elements of $\mathcal{G}_{k-1}(\Pi)$. We take any frame of $\Pi$ such that $S$ and $U$ are spanned by subsets of this frame. The associated apartment $\mathcal{A} \subset \mathcal{G}_{k}(\Pi)$ contains $X \in[S\rangle_{k}$ and $Y \in[U\rangle_{k}$ satisfying $d(X, Y) \geq 3$. Indeed, if the dimension of $S \cap U$ is less than $k-2$, then we choose any $X \in \mathcal{A} \cap[S\rangle_{k}$ and $Y \in \mathcal{A} \cap[U\rangle_{k}$ such that

$$
X \cap Y=S \cap U
$$

In the case when $S \cap U$ is $(k-2)$-dimensional we require in addition that every point of $X \backslash Y$ is non-collinear to a certain point of $Y$. See Lemma 1.

If $f_{k-1}(S)$ coincides with $f_{k-1}(U)$, then the intersection of $f(X)$ and $f(Y)$ is $\left(k^{\prime}-1\right)$-dimensional. By Lemma 1

$$
d(f(X), f(Y)) \leq 2
$$

which contradicts the fact that $f$ is an isometric embedding of $\Gamma_{k}(\Pi)$ in $\Gamma_{k^{\prime}}\left(\Pi^{\prime}\right)$.

For every $U \in \mathcal{G}_{k}(\Pi)$ we have

$$
f_{k-1}\left(\langle U]_{k-1}\right) \subset\langle f(U)]_{k^{\prime}-1},
$$


i.e., $f_{k-1}$ transfers tops to subsets of tops, which implies that $f_{k-1}$ sends adjacent vertices of $\Gamma_{k-1}(\Pi)$ to adjacent vertices of $\Gamma_{k^{\prime}-1}\left(\Pi^{\prime}\right)$. However, we cannot state that non-adjacent vertices of $\Gamma_{k-1}(\Pi)$ go to non-adjacent vertices of $\Gamma_{k^{\prime}-1}\left(\Pi^{\prime}\right)$.

Suppose that $k \geq 2$. Then $f_{k-1}$ transfers maximal cliques of $\Gamma_{k-1}(\Pi)$ (stars and tops) to subsets of maximal cliques of $\Gamma_{k^{\prime}-1}\left(\Pi^{\prime}\right)$. We do not show that $f_{k-1}$ is an isometric embedding of $\Gamma_{k-1}(\Pi)$ in $\Gamma_{k^{\prime}-1}\left(\Pi^{\prime}\right)$. It is sufficient to prove the following.

Lemma $6 f_{k-1}$ transfers stars to subsets of stars.

Proof Suppose that there exists a star $\mathcal{S} \subset \mathcal{G}_{k-1}(\Pi)$ whose image is not contained in a star of $\mathcal{G}_{k^{\prime}-1}\left(\Pi^{\prime}\right)$. Then $f_{k-1}(\mathcal{S})$ is a subset in a certain top $\mathcal{T} \subset \mathcal{G}_{k^{\prime}-1}\left(\Pi^{\prime}\right)$. We choose distinct $U_{1}, U_{2} \in \mathcal{G}_{k}(\Pi)$ such that for every $i=1,2$ the top $\left\langle U_{i}\right]_{k-1}$ intersects the star $\mathcal{S}$ in a line. Then the intersection of $\left\langle f\left(U_{i}\right)\right]_{k^{\prime}-1}$ and $\mathcal{T}$ contains more than one element. This is possible only in the case when the tops $\left\langle f\left(U_{i}\right)\right]_{k^{\prime}-1}$ and $\mathcal{T}$ are coincident. Hence $f\left(U_{1}\right)=f\left(U_{2}\right)$ which contradicts the fact that $f$ is injective.

Lemma 7 There is a sequence of injections

$$
f_{i}: \mathcal{G}_{i}(\Pi) \rightarrow \mathcal{G}_{k^{\prime}-k+i}\left(\Pi^{\prime}\right), \quad i=k, k-1, \ldots, 0
$$

such that $f_{k}=f$ and

$$
f_{i}\left([S\rangle_{i}\right) \subset\left[f_{i-1}(S)\right\rangle_{k^{\prime}-k+i}
$$

for every $S \in \mathcal{G}_{i-1}(\Pi)$ if $i \geq 1$.

Proof Using Lemmas 5, 6 and the arguments from the proof of Lemma 4 we show that $f_{k-1}$ transfers big stars to subsets of big stars and the image of every big star of $\mathcal{G}_{k-1}(\Pi)$ is contained in a unique big star of $\mathcal{G}_{k^{\prime}-1}\left(\Pi^{\prime}\right)$. So, $f_{k-1}$ induces a mapping

$$
f_{k-2}: \mathcal{G}_{k-2}(\Pi) \rightarrow \mathcal{G}_{k^{\prime}-2}\left(\Pi^{\prime}\right) \text {. }
$$

If $S$ and $U$ are distinct elements of $\mathcal{G}_{k-2}(\Pi)$, then there exist $X \in[S\rangle_{k}$ and $Y \in[U\rangle_{k}$ satisfying $d(X, Y) \geq 4$ (as in the proof of Lemma 5 we take a frame of $\Pi$ such that $S$ and $U$ are spanned by subsets of this frame and choose $X, Y$ in the associated apartment of $\left.\mathcal{G}_{k}(\Pi)\right)$. It is clear that

$$
f_{k-2}(S) \subset f(X) \text { and } f_{k-2}(U) \subset f(Y) .
$$

If $f_{k-2}(S)$ coincides with $f_{k-2}(U)$, then the dimension of the intersection of $f(X)$ and $f(Y)$ is not less than $k^{\prime}-2$. Lemma 1 shows that

$$
d(f(X), f(Y)) \leq 3
$$

and $f$ is not an isometric embedding of $\Gamma_{k}(\Pi)$ in $\Gamma_{k^{\prime}}\left(\Pi^{\prime}\right)$. Therefore, $f_{k-2}$ is injective.

The mapping $f_{k-2}$ transfers tops to subsets of tops. As in the proof of Lemma 6 we show that $f_{k-2}$ sends stars to subsets of stars if $k \geq 3$. Step by step, we construct the required sequence. 
The inclusion from Lemma 7 implies that

$$
f_{i-1}\left(\langle U]_{i-1}\right) \subset\left\langle f_{i}(U)\right]_{k^{\prime}-k+i-1}
$$

for every $U \in \mathcal{G}_{i}(\Pi)$. Also, we have

$$
f_{0}\left(\langle U]_{0}\right) \subset\left\langle f_{i}(U)\right]_{k^{\prime}-k}
$$

for all $U \in \mathcal{G}_{i}(\Pi)$ and $i \leq k$. Indeed, if $U \in \mathcal{G}_{i}(\Pi)$, then $\langle U]_{0}$ is the union of all $\langle S]_{0}$ such that $S \in\langle U]_{i-1}$ and the latter inclusion can be proved by induction.

Lemma 8 There exists $S \in \mathcal{G}_{k^{\prime}-k-1}\left(\Pi^{\prime}\right)$ such that the image of $f$ is contained in the big $\operatorname{star}[S\rangle_{k^{\prime}}$.

Proof The case when $k^{\prime}=k$ is trivial, and we suppose that $k^{\prime}>k$. The mapping $f_{0}$ is an injection sending lines of $\Pi$ to subsets in tops of $\mathcal{G}_{k^{\prime}-k}\left(\Pi^{\prime}\right)$. Hence it transfers collinear points of $\Pi$ to adjacent vertices of $\Gamma_{k^{\prime}-k}\left(\Pi^{\prime}\right)$. Let $M$ be a maximal singular subspace of $\Pi$. Then $f_{0}(M)$ is a clique of $\Gamma_{k^{\prime}-k}\left(\Pi^{\prime}\right)$. If $f_{0}(M)$ is contained in a top, then $f_{0}$ transfers all lines of $M$ to subsets of the same top which contradicts the fact that $f_{1}$ is injective. Thus $f_{0}(M)$ is a subset in a certain star and there exists $S \in \mathcal{G}_{k^{\prime}-k-1}\left(\Pi^{\prime}\right)$ such that

$$
f_{0}(M) \subset[S\rangle_{k^{\prime}-k}
$$

As in the proof of Proposition 1 (Sect. 4.2) we show that $f_{0}(p)$ belongs to $[S\rangle_{k^{\prime}-k}$ for every point $p \in P$. If $U \in \mathcal{G}_{k}(\Pi)$ and $p$ is a point of $U$, then

$$
S \subset f_{0}(p) \subset f(U)
$$

i.e., $f(U)$ belongs to $[S\rangle_{k^{\prime}}$.

Therefore $f$ is an isometric embedding of $\Gamma_{k}(\Pi)$ in $\Gamma_{k}\left(\Pi_{S}^{\prime}\right)$ and $f_{0}$ is an injection of the point set $P$ to $[S\rangle_{k^{\prime}-k}$ transferring lines of $\Pi$ to subsets in lines of $\Pi_{S}^{\prime}$.

Lemma 9 Let $U \in \mathcal{G}_{i}(\Pi)$ and $i \leq k$. If $U$ is spanned by points $p_{1}, \ldots, p_{i+1}$, then $f_{i}(U)$ is spanned by $f_{0}\left(p_{1}\right), \ldots, f_{0}\left(p_{i+1}\right)$.

Proof We prove the statement by induction. The case when $i=0$ is trivial. Suppose that $i \geq 1$, and consider the $(i-1)$-dimensional singular subspaces $M$ and $N$ spanned by $p_{1}, \ldots, p_{i}$ and $p_{2}, \ldots, p_{i+1}$, respectively. By the inductive hypothesis $f_{i-1}(M)$ and $f_{i-1}(N)$ are spanned by

$$
f_{0}\left(p_{1}\right), \ldots, f_{0}\left(p_{i}\right) \text { and } f_{0}\left(p_{2}\right), \ldots, f_{0}\left(p_{i+1}\right)
$$

respectively. The required statement follows from the fact that $f_{i-1}(M)$ and $f_{i-1}(N)$ are distinct $\left(k^{\prime}-k+i-1\right)$-dimensional singular subspaces contained in the $\left(k^{\prime}-k+i\right)$ dimensional singular subspace $f_{i}(U)$.

Our last step is the following. 
Lemma $10 f_{0}$ is a collinearity-preserving injection of $\Pi$ to $\Pi_{S}^{\prime}$.

Proof Since $f_{0}$ transfers lines of $\Pi$ to subsets in lines of $\Pi_{S}^{\prime}$, we need to show that $f_{0}$ sends non-collinear points of $\Pi$ to non-collinear points of $\Pi_{S}^{\prime}$.

Let $p$ and $q$ be non-collinear points of $\Pi$. Consider a frame

$$
p_{1}=p, p_{2}, \ldots, p_{n}, q_{1}=q, q_{2}, \ldots, q_{n},
$$

where every $p_{i}$ is non-collinear to $q_{i}$. Denote by $X$ and $Y$ the $k$-dimensional singular subspaces spanned by $p_{1}, \ldots, p_{k+1}$ and $q_{1}, \ldots, q_{k+1}$, respectively. Then

$$
d(X, Y)=k+2
$$

(see Lemma 1). By Lemma $9 f(X)$ and $f(Y)$ are $k$-dimensional subspaces of $\Pi_{S}^{\prime}$ spanned by

$$
f_{0}\left(p_{1}\right), \ldots, f_{0}\left(p_{k+1}\right) \text { and } f_{0}\left(q_{1}\right), \ldots, f_{0}\left(q_{k+1}\right),
$$

respectively. The point $p_{1}$ is collinear to $q_{i}$ if $i \neq 1$. Hence $f_{0}\left(p_{1}\right)$ and $f_{0}\left(q_{i}\right)$ are collinear points of $\Pi_{S}^{\prime}$ if $i \neq 1$. Therefore, if $f_{0}\left(p_{1}\right)$ is collinear to $f_{0}\left(q_{1}\right)$, then it is collinear to all points of $f(Y)$ which means that

$$
d(f(X), f(Y)) \leq k+1
$$

(see Lemma 1). The latter is impossible, since $f$ is an isometric embedding of $\Gamma_{k}(\Pi)$ in $\Gamma_{k^{\prime}}\left(\Pi^{\prime}\right)$. Thus $f_{0}(p)$ and $f_{0}(q)$ are non-collinear points of $\Pi_{S}^{\prime}$.

So, $f_{0}$ is a collinearity-preserving injection of $\Pi$ to $\Pi_{S}^{\prime}$. It follows from Lemma 9 that $f(U)$ coincides with $\left\langle f_{0}(U)\right\rangle$ for every $U \in \mathcal{G}_{k}(\Pi)$, i.e., $f$ is induced by $f_{0}$.

The rank of $\Pi_{S}^{\prime}$ is equal to $n^{\prime}-k^{\prime}+k$. The existence of collinearity-preserving injections of $\Pi$ to $\Pi_{S}^{\prime}$ implies that $n^{\prime}-k^{\prime}+k \geq n$. This completes the proof of Proposition 2.

\subsection{Proof of Theorem 1}

Let $f$ be as in the previous subsection. We need to show that $f$ transfers stars to subsets of stars if $n \geq 5$ and $k \leq n-4$.

Suppose that there is a star $\mathcal{S} \subset \mathcal{G}_{k}(\Pi)$ such that $f(\mathcal{S})$ is contained in a top of $\mathcal{G}_{k^{\prime}}\left(\Pi^{\prime}\right)$. If $X, Y, Z \in \mathcal{S}$ form a triangle, then their images form a top-triangle. The corresponding top is the unique maximal clique of $\Gamma_{k^{\prime}}\left(\Pi^{\prime}\right)$ containing $f(X), f(Y), f(Z)$. On the other hand, the singular subspace spanned by $X, Y, Z$ is $(k+2)$-dimensional. Since $k \leq n-4$, this singular subspace is not maximal. This guarantees the existence of a star $\mathcal{S}^{\prime}$ containing $X, Y, Z$ and different from $\mathcal{S}$. By the observation from Sect. 2.1 $f(\mathcal{S})$ and $f\left(\mathcal{S}^{\prime}\right)$ are subsets of distinct maximal cliques of $\Gamma_{k^{\prime}}\left(\Pi^{\prime}\right)$. Each of these cliques contains $f(X), f(Y), f(Z)$, and we get a contradiction. 


\section{Proof of Theorem 2}

In this section we suppose that $f$ is an isometric embedding of $\Gamma_{n-3}(\Pi)$ in $\Gamma_{k^{\prime}}\left(\Pi^{\prime}\right)$ and $n \geq 4$. By Sect. 4.3 we have $n-3 \leq k^{\prime} \leq n^{\prime}-3$.

\subsection{Regular pairs of triangles}

Let

$$
\Delta=\left\{S_{1}, S_{2}, S_{3}\right\} \text { and } \Delta^{\prime}=\left\{S_{1}^{\prime}, S_{2}^{\prime}, S_{3}^{\prime}\right\}
$$

be triangles in $\mathcal{G}_{k}(\Pi)$ such that $\Delta \cap \Delta^{\prime}=\emptyset$. We say that these triangles form a regular pair if $S_{i}$ and $S_{j}^{\prime}$ are adjacent vertices of $\Gamma_{k}(\Pi)$ only in the case when $i \neq j$; in other words, every vertex from each of these triangles is adjacent to precisely two vertices of the other triangle. In this case, one of the following possibilities is realized:

(1) There are $(k+2)$-dimensional singular subspaces $U$ and $U^{\prime}$ whose intersection $S$ is $(k-1)$-dimensional and $\Delta, \Delta^{\prime}$ are star-triangles contained in $[S, U]_{k}$ and $\left[S, U^{\prime}\right]_{k}$, respectively. Note that for every point $q \in U \backslash S$ there is a point of $U^{\prime}$ non-collinear to $q$. Similarly, for every point $q^{\prime} \in U^{\prime} \backslash S$ there is a point of $U$ non-collinear to $q^{\prime}$.

(2) One of the triangles is a star-triangle, and the other is a top-triangle. For example, if $\Delta$ is a star-triangle and $\Delta^{\prime}$ is a top-triangle, then the singular subspace $\left\langle S_{1}^{\prime}, S_{2}^{\prime}, S_{3}^{\prime}\right\rangle$ is $(k+1)$-dimensional and there is a point $p \notin\left\langle S_{1}^{\prime}, S_{2}^{\prime}, S_{3}^{\prime}\right\rangle$ collinear to all points of $\left\langle S_{1}^{\prime}, S_{2}^{\prime}, S_{3}^{\prime}\right\rangle$ and such that

$$
S_{1}=\left\langle p, S_{2}^{\prime} \cap S_{3}^{\prime}\right\rangle, S_{2}=\left\langle p, S_{1}^{\prime} \cap S_{3}^{\prime}\right\rangle, S_{3}=\left\langle p, S_{1}^{\prime} \cap S_{2}^{\prime}\right\rangle
$$

Note that all elements of our triangles are contained in the $(k+2)$-dimensional singular subspace spanned by $p$ and $\left\langle S_{1}^{\prime}, S_{2}^{\prime}, S_{3}^{\prime}\right\rangle$.

Indeed, two top-triangles do not form a regular pair. So, at least one of them is a star-triangle. If our triangles both are star-triangles, then

$$
S_{1} \cap S_{2} \cap S_{3}=S_{1}^{\prime} \cap S_{2}^{\prime} \cap S_{3}^{\prime}
$$

is a $(k-1)$-dimensional subspace, we denote it by $S$ and get (1). If one of the triangles is a star-triangle and the other is a top-triangle, then a direct verification shows that the case (2) is realized.

It is clear that $f$ transfers regular pairs of triangles to regular pairs of triangles.

\subsection{Proof of Theorem 2 for $n=4$}

Suppose that $n=4$. Then $f$ is an isometric embedding of $\Gamma_{1}(\Pi)$ in $\Gamma_{k^{\prime}}\left(\Pi^{\prime}\right)$. A maximal singular subspace $U \in \mathcal{G}_{3}(\Pi)$ is said to be special if there exists a point 
$p \in U$ such that $f$ transfers the star $[p, U]_{1}$ to a subset contained in a top. In the case when there exist no special maximal singular subspaces, we apply Proposition 2.

Lemma 11 If $U \in \mathcal{G}_{3}(\Pi)$ is special, then for every point $q \in U$ the image of $[q, U]_{1}$ is contained in a top and for every two-dimensional singular subspace $S \subset U$ the image of $\langle S]_{1}$ is a subset in a star.

This statement is proved for the case when $k^{\prime}=1$ and $n^{\prime}=4$ [13, Lemma 4.15]. Now we show that the same arguments work in the general case.

Proof Let $p$ be a point of $U$ such that $f\left([p, U]_{1}\right)$ is contained in a top

$$
\left\langle S^{\prime}\right]_{k^{\prime}}, \quad S^{\prime} \in \mathcal{G}_{k^{\prime}+1}\left(\Pi^{\prime}\right) .
$$

We take any two-dimensional singular subspace $S \subset U$ which does not contain the point $p$. Consider a regular pair of triangles

$$
\Delta \subset[p, U]_{1} \text { and } \Delta^{\prime} \subset\langle S]_{1} .
$$

Since $f(\Delta)$ is a top-triangle, the triangles $f(\Delta)$ and $f\left(\Delta^{\prime}\right)$ form a regular pair of type (2). Therefore $f\left(\Delta^{\prime}\right)$ is a star-triangle and $f\left(\langle S]_{1}\right)$ is contained in a certain star

$$
\left[T^{\prime}, U^{\prime}\right]_{k^{\prime}}, \quad T^{\prime} \in \mathcal{G}_{k^{\prime}-1}\left(\Pi^{\prime}\right), U^{\prime} \in \mathcal{G}_{n^{\prime}-1}\left(\Pi^{\prime}\right) .
$$

Note that $S^{\prime} \subset U^{\prime}$ and $T^{\prime} \not \subset S^{\prime}$.

Let $q$ be a point belonging to $U \backslash\{p\}$. We choose a two-dimensional singular subspace $S \subset U$ which does not contain $p$ and $q$. It was established above that $f\left(\langle S]_{1}\right)$ is a subset in a certain star (1). Consider a regular pair of triangles

$$
\Delta \subset[q, U]_{1} \text {, and } \Delta^{\prime} \subset\langle S]_{1}
$$

Then $q \notin S$ and $f\left(\Delta^{\prime}\right)$ is a star-triangle. Suppose that the triangles $f(\Delta)$ and $f\left(\Delta^{\prime}\right)$ form a regular pair of type (1). Then $f\left([q, U]_{1}\right)$ is contained in $\left[T^{\prime}, U^{\prime \prime}\right]_{k^{\prime}}$ for a certain $U^{\prime \prime} \in \mathcal{G}_{n^{\prime}-1}\left(\Pi^{\prime}\right)$. Since $T^{\prime} \not \subset S^{\prime}$, the top $\left\langle S^{\prime}\right]_{k^{\prime}}$ and the star $\left[T^{\prime}, U^{\prime \prime}\right]_{k^{\prime}}$ are disjoint. On the other hand,

$$
f\left([p, U]_{1}\right) \subset\left\langle S^{\prime}\right]_{k^{\prime}}, \quad f\left([q, U]_{1}\right) \subset\left[T^{\prime}, U^{\prime \prime}\right]_{k^{\prime}}
$$

and the stars $[p, U]_{1},[q, U]_{1}$ both contain the line joining $p$ and $q$. This contradiction shows that $f(\Delta)$ and $f\left(\Delta^{\prime}\right)$ form a regular pair of type (2), i.e., $f(\Delta)$ is a top-triangle. Then the image of $[q, U]_{1}$ is contained in a top.

Let $S$ be a two-dimensional singular subspace of $U$ containing the point $p$. We take any point $q \in U \backslash S$. Then $f\left([q, U]_{1}\right)$ is contained in a top. As above, we establish that $f\left(\langle S]_{1}\right)$ is a subset in a star. 
Lemma 12 Let $U, Q \in \mathcal{G}_{3}(\Pi)$. If $U$ is special and $\operatorname{dim}(U \cap Q)=2$, then $Q$ is not special.

Proof We take two distinct lines $L_{1}, L_{2} \subset U \cap Q$ and consider star-triangles

$$
\Delta=\left\{L_{1}, L_{2}, L_{3}\right\} \text { and } \Delta^{\prime}=\left\{L_{1}, L_{2}, L_{3}^{\prime}\right\}
$$

contained in $\langle U]_{1}$ and $\langle Q]_{1}$, respectively. The lines $L_{3}$ and $L_{3}^{\prime}$ have a common point; on the other hand, they contain non-collinear points which means that the distance between them in $\Gamma_{1}(\Pi)$ is equal to 2 . Thus

$$
d\left(f\left(L_{3}\right), f\left(L_{3}^{\prime}\right)\right)=2 .
$$

By our assumption, $f(\Delta)$ is a top-triangle. If the same holds for $f\left(\Delta^{\prime}\right)$, then $f\left(L_{3}^{\prime}\right)$ belongs to the top containing $f(\Delta)$, i.e., $f\left(L_{3}^{\prime}\right)$ is contained in the $\left(k^{\prime}+1\right)$-dimensional subspace spanned by $f\left(L_{1}\right), f\left(L_{2}\right), f\left(L_{3}\right)$. This implies that $f\left(L_{3}\right)$ and $f\left(L_{3}^{\prime}\right)$ are adjacent vertices of $\Gamma_{k^{\prime}}\left(\Pi^{\prime}\right)$ and we get a contradiction. So, $f\left(\Delta^{\prime}\right)$ is a star-triangle. Then $f$ transfers the star containing $\Delta^{\prime}$ to a subset in a star. By Lemma $11, Q$ is not special.

Lemma 13 Let $U, Q \in \mathcal{G}_{3}(\Pi)$. If $U$ is special and $\operatorname{dim}(U \cap Q)=1$, then $Q$ is special.

Proof The intersection of $U$ and $Q$ is a line. We denote this line by $L$. Consider the star-triangles

$$
\Delta=\left\{L, L_{1}, L_{2}\right\} \subset\langle U]_{1} \text { and } \Delta^{\prime}=\left\{L, L_{1}^{\prime}, L_{2}^{\prime}\right\} \subset\langle Q]_{1}
$$

such that $L_{i}$ and $L_{j}^{\prime}$ are adjacent vertices of $\Gamma_{1}(\Pi)$ only in the case when $i=j$. Then $f(\Delta)$ is a top-triangle. We observe that

$$
f(L), f\left(L_{1}\right), f\left(L_{1}^{\prime}\right)
$$

form a triangle. This is not a top-triangle (otherwise, $f\left(L_{1}^{\prime}\right)$ belongs to the top containing $f(\Delta)$ which is impossible). Therefore $f\left(L_{1}^{\prime}\right)$ contains the $\left(k^{\prime}-1\right)$-dimensional singular subspace $f(L) \cap f\left(L_{1}\right)$. Similarly, we establish that

$$
f(L), f\left(L_{2}\right), f\left(L_{2}^{\prime}\right)
$$

form a star-triangle and $f\left(L_{2}^{\prime}\right)$ contains the $\left(k^{\prime}-1\right)$-dimensional singular subspace $f(L) \cap f\left(L_{2}\right)$. Since $f(\Delta)$ is a top-triangle, we have

$$
f(L) \cap f\left(L_{1}\right) \neq f(L) \cap f\left(L_{2}\right) .
$$

So, $f\left(L_{1}^{\prime}\right)$ and $f\left(L_{2}^{\prime}\right)$ intersect $f(L)$ in two distinct $\left(k^{\prime}-1\right)$-dimensional singular subspaces which means that $f\left(\Delta^{\prime}\right)$ is a top-triangle. Then $f$ sends the star containing $\Delta^{\prime}$ to a subset in a top, i.e., $Q$ is special. 
Let $X$ and $Y$ be adjacent vertices of $\Gamma_{3}(\Pi)$. Suppose that $\Pi$ is a polar space of type $\mathrm{C}_{4}$. Then the line joining $X$ and $Y$ contains a certain $Z \in \mathcal{G}_{3}(\Pi)$ distinct from $X, Y$. We take any $T \in \mathcal{G}_{3}(\Pi)$ which does not belong to this line and such that $Z, T$ are adjacent vertices of $\Gamma_{3}(\Pi)$. Then

$$
\operatorname{dim}(X \cap T)=\operatorname{dim}(Y \cap T)=1 .
$$

If $X$ is special, then $T$ is special by Lemma 13 . We apply Lemma 13 to $T, Y$ and establish that $Y$ is special. The latter is impossible by Lemma 12.

Therefore the existence of special maximal singular subspaces implies that $\Pi$ is a polar space of type $D_{4}$. Also, it follows from Lemmas 12 and 13 that all special maximal singular subspaces form one of the half-spin Grassmannians $\mathcal{G}_{\delta}(\Pi), \delta \in\{+,-\}$. Every two-dimensional singular subspace of $\Pi$ is contained in a certain element of $\mathcal{G}_{\delta}(\Pi)$. Thus $f$ transfers every top to a subset in a star. Let $g$ be the automorphism of $\Gamma_{1}(\Pi)$ described in Sect. 2.5. Then the composition $f g$ is an isometric embedding of $\Gamma_{1}(\Pi)$ in $\Gamma_{k^{\prime}}\left(\Pi^{\prime}\right)$ transferring stars to subsets of stars. We apply Proposition 2 again.

\subsection{Proof of Theorem 2 for $n \geq 5$}

Let $n \geq 5$. Suppose that there is a star $[S, U]_{n-3}$ in $\mathcal{G}_{n-3}(\Pi)$ such that $f\left([S, U]_{n-3}\right)$ is contained in a top. The singular subspace $S$ is $(n-4)$-dimensional, and we take any (n-5)-dimensional singular subspace $T \subset S$. The rank of the polar space $\Pi_{T}$ is equal to 4 , and $\mathcal{G}_{1}\left(\Pi_{T}\right)$ coincides with $[T\rangle_{n-3}$. The star $[S, U]_{n-3}$ is contained in $[T\rangle_{n-3}$, i.e., it is a star of $\mathcal{G}_{1}\left(\Pi_{T}\right)$. The restriction of $f$ to $[T\rangle_{n-3}$ is an isometric embedding of $\Gamma_{1}\left(\Pi_{T}\right)$ in $\Gamma_{k^{\prime}}\left(\Pi^{\prime}\right)$. By Sect. 5.2, $\Pi_{T}$ is a polar space of type $D_{4}$ which implies that $\Pi$ is a polar space of type $D_{n}$. Thus $f$ transfers stars to subsets of stars if $\Pi$ is a polar space of type $\mathrm{C}_{n}$. As above, we use Proposition 2.

Now we consider the case when $k^{\prime}=n^{\prime}-3$ and show that $f$ sends every star to a subset in a star.

Suppose that $\mathcal{S} \subset \mathcal{G}_{n-3}(\Pi)$ is a star such that $f(\mathcal{S})$ is contained in a top. We take any top

$$
\langle U]_{n-3}, \quad U \in \mathcal{G}_{n-2}(\Pi)
$$

intersecting $\mathcal{S}$ in a line. Since the intersection of two distinct tops contains at most one element, $f\left(\langle U]_{n-3}\right)$ cannot be in a top. Hence it is contained in a certain star

$$
\left[S^{\prime}, U^{\prime}\right]_{n^{\prime}-3}, \quad S^{\prime} \in \mathcal{G}_{n^{\prime}-4}\left(\Pi^{\prime}\right), U^{\prime} \in \mathcal{G}_{n^{\prime}-1}\left(\Pi^{\prime}\right)
$$

If $X_{1}, X_{2}, X_{3} \in\langle U]_{n-3}$ form a triangle, then their images form a star-triangle and

$$
\left\langle f\left(X_{1}\right), f\left(X_{2}\right), f\left(X_{3}\right)\right\rangle=U^{\prime}
$$

The dimension of

$$
S:=X_{1} \cap X_{2} \cap X_{3}
$$


is equal to $n-5$. We choose $Y \in \mathcal{G}_{n-3}(\Pi)$ satisfying the following conditions:

- $Y \cap U=S$,

- There is a point of $Y \backslash U$ collinear to all points of $U$ (note that the singular subspace $U$ is not maximal).

By Lemma $1, d\left(X_{i}, Y\right)=2$ for every $i$ and we have $d(Z, Y)=3$ for every $Z \in\langle U]_{n-3}$ which does not contain $S$. We want to show that

$$
d\left(X^{\prime}, f(Y)\right) \leq 2 \quad \forall X^{\prime} \in\left[S^{\prime}, U^{\prime}\right]_{n^{\prime}-3} .
$$

This contradicts the fact that $f$ is an isometric embedding of $\Gamma_{n-3}(\Pi)$ in $\Gamma_{n^{\prime}-3}\left(\Pi^{\prime}\right)$, and we get the claim.

For every $i \in\{1,2,3\}$ we have

$$
d\left(f\left(X_{i}\right), f(Y)\right)=2
$$

and, by Lemma 1, the dimension of every $f\left(X_{i}\right) \cap f(Y)$ is equal to $n^{\prime}-4$ or $n^{\prime}-5$.

First, we consider the case when $f\left(X_{i}\right) \cap f(Y)$ is $\left(n^{\prime}-4\right)$-dimensional for a certain $i$. If $f\left(X_{i}\right) \cap f(Y)$ coincides with $S^{\prime}$, then $f(Y)$ belongs to the big $\operatorname{star}\left[S^{\prime}\right\rangle_{n^{\prime}-3}$ which implies (2). If $f\left(X_{i}\right) \cap f(Y)$ is distinct from $S^{\prime}$, then

$$
\operatorname{dim}\left(f(Y) \cap S^{\prime}\right)=n^{\prime}-5
$$

and there is a point

$$
p \in\left(f\left(X_{i}\right) \cap f(Y)\right) \backslash S^{\prime}
$$

This point belongs to $U^{\prime}$. Hence $p \in f(Y) \backslash S^{\prime}$ is collinear to all points of every element of $\left[S^{\prime}, U^{\prime}\right]_{n^{\prime}-3}$. Since $f(Y) \cap S^{\prime}$ is $\left(n^{\prime}-5\right)$-dimensional, we get (2) again (see Lemma 1).

Now we suppose that $f\left(X_{i}\right) \cap f(Y)$ is $\left(n^{\prime}-5\right)$-dimensional for every $i$. If this subspace is contained in $S^{\prime}$ for a certain $i$, then all $f\left(X_{i}\right) \cap f(Y)$ are coincident with $f(Y) \cap S^{\prime}$ and the latter subspace is $\left(n^{\prime}-5\right)$-dimensional. Since the distance between $f\left(X_{i}\right)$ and $f(Y)$ is equal to 2 , for every $i$ there exists a point

$$
p_{i} \in f\left(X_{i}\right) \backslash f(Y)
$$

collinear to all points of $f(Y)$ (see Lemma 1). If one of the points $p_{i}$ belongs to $S^{\prime}$, then it is contained in every element of $\left[S^{\prime}, U^{\prime}\right]_{n^{\prime}-3}$. This implies (2), since $f(Y) \cap S^{\prime}$ is $\left(n^{\prime}-5\right)$-dimensional (see Lemma 1). Suppose that $p_{i} \in f\left(X_{i}\right) \backslash S^{\prime}$ for every $i$, and consider the two-dimensional singular subspace $T$ spanned by $p_{1}, p_{2}, p_{3}$ (the dimension of $T$ is equal to 2 , since the $\left(n^{\prime}-1\right)$-dimensional singular subspace $U^{\prime}$ is spanned by $T$ and the $\left(n^{\prime}-4\right)$-dimensional singular subspace $\left.S^{\prime}\right)$. Every point of $T$ is collinear to all points of $f(Y)$ and $T \cap S^{\prime}=\emptyset$. Each $X^{\prime} \in\left[S^{\prime}, U^{\prime}\right]_{n^{\prime}-3}$ has a non-empty intersection with $T$, i.e., there is a point of $X^{\prime} \backslash S^{\prime}$ collinear to all points of $f(Y)$. As above, we get (2). 
Consider the case when every $f\left(X_{i}\right) \cap f(Y)$ is not contained in $S^{\prime}$. In this case, the singular subspace $f(Y) \cap S^{\prime}$ is $\left(n^{\prime}-6\right)$-dimensional. We choose three points

$$
p_{i} \in\left(f\left(X_{i}\right) \cap f(Y)\right) \backslash S^{\prime}, \quad i=1,2,3 .
$$

They span a two-dimensional singular subspace. Then the singular subspace spanned by $f(Y) \cap S^{\prime}$ and $p_{1}, p_{2}, p_{3}$ is $\left(n^{\prime}-3\right)$-dimensional. Hence it coincides with $f(Y)$. This means that $f(Y)$ is contained in $U^{\prime}$. Since the dimension of the intersection of two $\left(n^{\prime}-3\right)$-dimensional subspaces of $U^{\prime}$ is not less than $n^{\prime}-5$, we have (2).

Remark 3 The condition $k^{\prime}=n^{\prime}-3$ is exploited in the latter paragraph only. If $k^{\prime}<n^{\prime}-3$, then we cannot state that the dimension of the intersection of two $k^{\prime}-$ dimensional subspaces of a maximal singular subspace is not less than $k^{\prime}-2$. So, we cannot prove (2).

\section{Proof of Theorem 3}

Suppose that $n=n^{\prime}$ and $f$ is an isometric embedding of $\Gamma_{n-2}(\Pi)$ in $\Gamma_{n-2}\left(\Pi^{\prime}\right)$. Maximal cliques of the graphs $\Gamma_{n-2}(\Pi)$ and $\Gamma_{n-2}\left(\Pi^{\prime}\right)$ are tops, and $f$ transfers every top to a subset contained in a top, i.e., there exists a mapping

$$
g: \mathcal{G}_{n-1}(\Pi) \rightarrow \mathcal{G}_{n-1}\left(\Pi^{\prime}\right)
$$

such that

$$
f\left(\langle U]_{n-2}\right) \subset\langle g(U)]_{n-2}
$$

for every $U \in \mathcal{G}_{n-1}(\Pi)$. The mapping $g$ is injective (distinct maximal cliques go to subsets of distinct maximal cliques).

Two distinct tops of $\mathcal{G}_{n-2}(\Pi)$ have a non-empty intersection if and only if the corresponding elements of $\mathcal{G}_{n-1}(\Pi)$ are adjacent vertices of $\Gamma_{n-1}(\Pi)$. The same holds for tops of $\mathcal{G}_{n-2}\left(\Pi^{\prime}\right)$. This implies that $g$ sends adjacent vertices of $\Gamma_{n-1}(\Pi)$ to adjacent vertices of $\Gamma_{n-1}\left(\Pi^{\prime}\right)$. Therefore

$$
d(X, Y) \geq d(g(X), g(Y))
$$

for all $X, Y \in \mathcal{G}_{n-1}(\Pi)$. We want to show that $g$ is an isometric embedding of $\Gamma_{n-1}(\Pi)$ in $\Gamma_{n-1}\left(\Pi^{\prime}\right)$. Since every geodesic in $\Gamma_{n-1}(\Pi)$ can be extended to a maximal geodesic consisting of $n$ edges, it is sufficient to establish that for any $X, Y \in \mathcal{G}_{n-1}(\Pi)$ satisfying $d(X, Y)=n$ we have

$$
d(f(X), f(Y))=n
$$

Let $X, Y \in \mathcal{G}_{n-1}(\Pi)$ and $d(X, Y)=n$. Consider a frame $p_{1}, \ldots, p_{n}, q_{1}, \ldots, q_{n}$ such that every $p_{i}$ is non-collinear to $q_{i}$ and

$$
X=\left\langle p_{1}, \ldots, p_{n}\right\rangle, \quad Y=\left\langle q_{1}, \ldots, q_{n}\right\rangle
$$


Then

$$
A:=\left\langle p_{1}, \ldots, p_{n-1}\right\rangle, \quad B:=\left\langle p_{2}, \ldots, p_{n}\right\rangle
$$

and

$$
C:=\left\langle q_{1}, \ldots, q_{n-1}\right\rangle, \quad D:=\left\langle q_{2}, \ldots, q_{n}\right\rangle
$$

are elements of $\mathcal{G}_{n-2}(\Pi)$ satisfying the following conditions:

(1) $X=\langle A, B\rangle$ and $Y=\langle C, D\rangle$,

(2) $d(A, C)=d(B, D)=n$ and $d(A, D)=d(B, C)=n-1$.

We have

$$
g(X)=\langle f(A), f(B)\rangle, \quad g(Y)=\langle f(C), f(D)\rangle
$$

and

$$
\begin{aligned}
& d(f(A), f(C))=d(f(B), f(D))=n, \\
& d(f(A), f(D))=d(f(B), f(C))=n-1 .
\end{aligned}
$$

Suppose that the distance between $g(X)$ and $g(Y)$ is less than $n$. Then $g(X)$ and $g(Y)$ have a non-empty intersection.

Lemma 14 No point of $g(X) \cap g(Y)$ belongs to $f(A) \cup f(B) \cup f(C) \cup f(D)$.

Proof Since the distance between $f(A)$ and $f(C)$ is equal to $n$, Lemma 1 shows that $f(A) \cap f(C)=\emptyset$ and every point of $f(A)$ is non-collinear to a certain point of $f(C)$. On the other hand, if $p \in g(X) \cap g(Y)$, then it is collinear to all points of $f(C)$. Hence $p$ does not belong to $f(A)$. Similarly, we show that $p$ does not belong to any of $f(B), f(C), f(D)$.

The distance between $f(A)$ and $f(D)$ is equal to $n-1$ and, by Lemma 1 , we have two possibilities. In each of these cases, there is a point $q \in f(D)$ collinear to all points of $f(A)$. Let $p \in g(X) \cap g(Y)$. By Lemma $14 g(X)$ is spanned by $f(A)$ and $p$. Since $q$ is collinear to $p$ and all points of $f(A)$, it is collinear to all points of $g(X)$. The latter means that $q \in g(X)$ (recall that $g(X)$ is a maximal singular subspace of $\left.\Pi^{\prime}\right)$. Then $q$ belongs to $g(X) \cap g(Y)$, which contradicts Lemma 14 .

Thus $g(X) \cap g(Y)=\emptyset$, i.e., the distance between $g(X)$ and $g(Y)$ is equal to $n$. So, $g$ is an isometric embedding of $\Gamma_{n-1}(\Pi)$ in $\Gamma_{n-1}\left(\Pi^{\prime}\right)$. By [14] it is induced by a collinearity-preserving injection from $\Pi$ to $\Pi^{\prime}$. If $S \in \mathcal{G}_{n-2}(\Pi)$ is the intersection of $X, Y \in \mathcal{G}_{n-1}(\Pi)$, then $f(S)$ coincides with $g(X) \cap g(Y)$. This implies that $f$ is induced by the same collinearity-preserving injection of $\Pi$ to $\Pi^{\prime}$.

Remark 4 The above arguments do not work for the case when $f$ is an isometric embedding of $\Gamma_{n-2}(\Pi)$ in $\Gamma_{n^{\prime}-2}\left(\Pi^{\prime}\right)$ and $n<n^{\prime}$. If $A, B \in \mathcal{G}_{n-2}(\Pi)$ and $d(A, B)=$ $n$, then the distance between $f(A)$ and $f(B)$ is not maximal in $\Gamma_{n^{\prime}-2}\left(\Pi^{\prime}\right)$. Lemma 1 shows that there are two possibilities for the dimension of $f(A) \cap f(B)$ and always there is a point of $f(A)$ collinear to all points of $f(B)$. 
Open Access This article is distributed under the terms of the Creative Commons Attribution 4.0 International License (http://creativecommons.org/licenses/by/4.0/), which permits unrestricted use, distribution, and reproduction in any medium, provided you give appropriate credit to the original author(s) and the source, provide a link to the Creative Commons license, and indicate if changes were made.

\section{References}

1. Blok, R.J., Cooperstein, B.N.: Projective subgrassmannians of polar grassmannians. Bull. Belg. Math. Soc. Simon Stevin 17, 675-691 (2010)

2. Buekenhout, F., Cohen, A.M.: Diagram Geometry. Ergebnisse der Mathematik und ihrer Grenzgebiete (Results in Mathematics and Related Areas) 57. Springer, Heidelberg (2013)

3. Chow, W.L.: On the geometry of algebraic homogeneous spaces. Ann. Math. 50, 32-67 (1949)

4. Cooperstein, B.N., Kasikova, A., Shult, E.E.: Witt-type theorems for Grassmannians and Lie incidence geometries. Adv. Geom. 5, 15-36 (2005)

5. Cooperstein, B.N.: Classical subspaces of symplectic Grassmannians. Bull. Belg. Math. Soc. Simon Stevin 12, 719-725 (2005)

6. Cooperstein, B.N.: Symplectic subspaces of symplectic Grassmannians. Eur. J. Comb. 28, 1442-1454 (2007)

7. Cooperstein, B.N.: Witt-Type Theorems for Subspaces of Lie Geometries: A Survey. In: Groups of Exceptional Type, Coxeter Groups and Related Geometries. Springer Proceedings in Mathematics and Statistics, vol. 82, pp. 123-133 (2014)

8. Deza, M., Deza, E.: Encyclopedia of Distances, 3rd edn. Springer, New York (2014)

9. Dieudonné, J.: La géométrie des Groupes Classiques. Springer, New York (1971)

10. Kasikova, A.: Characterization of some subgraphs of point-collinearity graphs of building geometries. Eur. J. Comb. 28, 1493-1529 (2007)

11. Kasikova, A.: Characterization of some subgraphs of point-collinearity graphs of building geometries II. Adv. Geom. 9, 45-84 (2009)

12. Kasikova, A.: A characterization of point shadows of residues in building geometries. Innov. Incid. Geom. 13, 179-206 (2013)

13. Pankov M.: Grassmannians of Classical Buildings, Algebra and Discrete Math. Series 2. World Scientific, Singapore (2010)

14. Pankov, M.: Metric characterization of apartments in dual polar spaces. J. Comb. Theory Ser. A 118, 1313-1321 (2011)

15. Pankov, M.: Characterization of apartments in polar Grassmannians. Bull. Belg. Math. Soc. Simon Stevin 19, 345-366 (2012)

16. Pankov M.: Isometric embeddings of half-cube graphs in half-spin Grassmannians. Electron. J. Comb. 21, P4.4 (2014)

17. Pankov, M.: Geometry of Semilinear Embeddings: Relations to Graphs and Codes. World Scientific, Singapore (2015)

18. Pasini, A.: Diagram Geometries. Oxford Science Publications, Oxford (1994)

19. Shult, E.E.: Points and Lines. Characterizing the Classical Geometries, Universitext XXII. Springer, Berlin (2011)

20. Tits, J.: Buildings of Spherical Type and Finite BN-Pairs. Lecture Notes in Mathematics 386, Springer, New York (1974)

21. Ueberberg, J.: Foundations of incidence geometry. Projective and Polar Spaces, Springer Monographs in Mathematics. Springer, Heidelberg (2011) 